Research paper

\title{
Longitudinal monitoring of heartbeat dynamics predicts mood changes in bipolar patients: A pilot study
}

\author{
Claudio Gentili ${ }^{\mathrm{a}, *}$, Gaetano Valenza ${ }^{\mathrm{b}}$, Mimma Nardelli ${ }^{\mathrm{b}}$, Antonio Lanatà ${ }^{\mathrm{b}}$, Gilles Bertschy ${ }^{\mathrm{c}}$, \\ Luisa Weiner ${ }^{\mathrm{c}}$, Mauro Mauri ${ }^{\mathrm{d}}$, Enzo Pasquale Scilingo ${ }^{\mathrm{b}}$, Pietro Pietrini ${ }^{\mathrm{e}, *}$ \\ a Department of General Psychology, University of Padua, Via Venezia 8, 35139 Padua, Italy \\ b Department of Information Engineering \& Research Centre "E. Piaggio", School of Engineering, University of Pisa, Italy \\ ${ }^{\mathrm{c}}$ INSERM U1114, Fédération de Médecine Translationnelle de Strasbourg, Université de Strasbourg, Pôle de Psychiatrie et Santé Mentale des Hôpitaux \\ Universitaires de Strasbourg, France \\ d Section of Psychiatry, Department of Clinical and Experimental Medicine, University of Pisa, Italy \\ e IMT School for Advanced Studies, Piazza San Ponziano, 6 - 55100 Lucca, Italy
}

\section{A R T I C L E I N F O}

\section{Keywords:}

Bipolar disorders

Heart rate variability

Psychophysiology

Biological psychiatry

Supported vector machine

\begin{abstract}
A B S T R A C T
Objectives: Recent research indicates that Heart Rate Variability (HRV) is affected in Bipolar Disorders (BD) patients. To determine whether such alterations are a mere expression of the current mood state or rather contain longitudinal information on BD course, we examined the potential influence of states adjacent in time upon HRV features measured in a target mood state.

Methods: Longitudinal evaluation of HRV was obtained in eight BD patients by using a wearable monitoring system developed within the PSYCHE project. We extracted time-domain, frequency-domain and non-linear HRV-features and trained a Support Vector Machine (SVM) to classify HRV-features according to mood state. To evaluate the influence of adjacent mood states, we trained SVM with different HRV-feature sets: 1) belonging to each mood state considered alone; 2) belonging to each mood state and normalized using information from the preceding mood state; 3) belonging to each mood state and normalized using information from the preceding and subsequent mood states; 4) belonging to each mood state and normalized using information from two randomly chosen states.

Results: SVM classification accuracy within a target state was significantly greater when HRV-features from the previous and subsequent mood states were considered.

Conclusions: Although preliminary and in need of replications our results suggest for the first time that psychophysiological states in BD contain information related to the subsequent ones. Such characteristic may be used to improve clinical management and to develop algorithms to predict clinical course and mood switches in individual patients.
\end{abstract}

\section{Introduction}

In contrast to all the other branches of medicine, psychiatry has suffered since birth from a lack of external indices to validate the diagnosis and to monitor the clinical course of disorders. With the exception of psychiatric conditions caused by a primary somatic disorder (e.g., Major Depression due to hypothyroidism), no biological marker to date has proven to be reliable, consistent and accurate enough to be used in practice for clinical (Antonijevic, 2008; Ozerdema et al., 2013; Teixeira et al., 2013) as well as forensic purposes (Sartori et al., 2011). Specifically, while sleep alterations (e.g., REM latency) (Antonijevic, 2008), hormonal changes (e.g., cortisol rhythm altera- tions) (Antonijevic, 2008; Teixeira et al., 2013), and brain metabolic (e.g. metabolism reductions in the prefrontal cortex) (Ozerdema et al., 2013) or structural alterations (e.g., reductions in hippocampal volume) (Arnone et al., 2012; McKinnon et al., 2009) have been found to be altered in mood disorders, none of these changes has shown enough sensitivity or specificity to make any of them feasible for a clinical use.

Recently, the study of the Autonomic Nervous System (ANS) has provoked a growing interest in the assessment of both psychiatric as well as somatic disorders. Significant alterations in heartbeat dynamics, including alterations in the Heart Rate Variability (HRV) which is the complex modification of the heart rate over time - have been detected in several somatic and mental disorders (Alvares et al.,

\footnotetext{
* Corresponding authors.

E-mail addresses: c.gentili@unipd.it (C. Gentili), pietro.pietrini@imtlucca.it (P. Pietrini).
} 
2016; Henry et al., 2010; Rajendra Acharya et al., 2006). A recent meta-analysis, for instance, has shown a consistent reduction of different HRV measures in patients with psychiatric disorders as compared to controls (Alvares et al., 2016). Such decrease in HRV was smaller for anxiety disorders and maximal in psychotic patients. It is important to note that this meta-analysis found such a consistent reduction by grouping different measures of HRV. As a matter of fact, HRV analysis does not provide a single index, but a series of features that describe different and independent information. HRV can be characterized using distinct methods, which assess it either in time or in frequency domains (Henry et al., 2010; Rajendra Acharya et al., 2006). In particular, frequency domain methods consider two components of the variability, the low (Low Frequency -LF, 0.04-0.15 Hz) and the high (High Frequency - HF, 0.15-0.4 Hz) frequencies. The HF component of the HRV is basically determined by parasympathetic activity (Rajendra Acharya et al., 2006), while LF is affected by both parasympathetic and sympathetic modulation. In fact, components above $0.15 \mathrm{~Hz}$ (i.e., HF band) seem to be exclusively mediated by vagal activity (Henry et al., 2010; Rajendra Acharya et al., 2006), where the strongest contribution to HF power modulation is due to respiration (the so-called Respiratory Sinus Arrhythmia). On the other hand, changes below $0.15 \mathrm{~Hz}$ (i.e., LF band) are mediated by both cardiac vagal and sympathetic nerves with the contribution of other hemodynamic- and vascular-mediated modulations (Saul et al., 1991), including baroreflex activity (Goldstein et al., 2011; Rahman et al., 2011). For this reason, it has been proposed that the $\mathrm{LF} / \mathrm{HF}$ ratio may be a valid index of the sympatho-vagal balance (Montano et al., 2009). However, given the fact that LF is mediated both by vagal and sympathetic innervations, and by the previously mentioned other hemodynamicand vascular-mediated modulations, both the exact physiological meaning of LF component and the reliability of the LF/HF ratio as a measure of sympatho-vagal balance are controversial (Goldstein et al., 2011; Heathers, 2012; Rahman et al., 2011). In fact, recent reports suggested that the main SNA component of LF is parasympathetic as well (McCraty and Shaffer, 2015; Reyes del Paso et al., 2013). Studies on both physiological (e.g., physical exercise (Warren et al., 1997)) and pharmacological (e.g. adrenergic agonists (Ahmed et al., 1994)) sympathetic stimulation failed to show the expected LF power increases and, more in general, the published findings are far less consistent than one can glance at a first sight (Reyes del Paso et al., 2013).

Another way to assess HRV is by measuring its nonlinear domains, including complexity and fractal features (Rajendra Acharya et al., 2006). Analysis methods derived from nonlinear system dynamics have started a novel approach for studying the characteristics of HRV. These methods differ from the conventional ones because they are designed to assess the quality, scaling and correlation properties of the signals (Aubert et al., 2009). Although nonlinear analyses are more difficult to interpret in terms of physiological meaning, it has been suggested that "Nonlinear control of HRV poses potential physiological advantages in the possibility of adapting quickly and more subtly to changes in physiological needs" (Aubert et al., 2009). Although some authors highlighted some shortcomings in the use of this type of features, including some reproducibility matters (Tan et al., 2009), they have been suggested to have some valid prognostic and diagnostic uses (e.g., (Leistedt et al., 2011; Yang et al., 2013; Yang and Tsai, 2013)).

HRV alterations have been found in cardiac disorders, including coronary disease and heart failure (Feng et al., 2014; Harris et al., 2014; Peterson et al., 2014; Radaelli et al., 2014; Sandercock and Brodie, 2006; Wendt et al., 2014). Particularly, HRV alterations may predict prognosis and can be used as a marker of the health of the heart (Sandercock and Brodie, 2006). The most frequent findings are reduced variability, changes in the HRV spectral distribution and a reduction in HRV complexity. Thus, in general, a loss of variability or a prevalence of the LF component of the spectrum are associated with a more compromised heart function and a poorer prognosis (Sandercock and Brodie, 2006). On the contrary, an increased HRV is associated with a better health not only in patients but also in healthy volunteers, so as to be considered a valuable indicator of general well-being (Gonçalves et al., 2014). Given this data, it is not surprising that other studies have shown that HRV can be used as a marker to assess the severity of distinct clinical conditions, including stroke (Tang et al., 2015), epilepsy (Lotufo et al., 2012), multi-organ failures (Liu et al., 2013; Zhang et al., 2015), cancer fatigue (Crosswell et al., 2014) and autoimmune diseases (Adlan et al., 2014; Holman and Ng, 2008).

Regarding mood disorders, several indications support a link between heart function and mood: a) There is a frequent occurrence of comorbidity between mood disorders (particularly, but not only, Major Depression) and cardiovascular conditions (particularly, but not only, coronary artery diseases) (Jiang, 2008; Lichtman et al., 2014; Rovai et al., 2015); b) occurrence of mood disorders in patients with heart diseases is associated with a worse clinical prognosis (Lichtman et al., 2014); c) presence of cardiovascular disorders significantly increases the risk of developing a Mood Disorder (Jiang, 2008); d) a common biological milieu has been proposed for such a frequent comorbidity and HRV alterations can be part of it (Rovai et al., 2015).

Thus, it is not surprising to find research on autonomic nervous system and its alterations in relation to psychopathology. HRV alterations have been found in acute and chronic stressful conditions (Jones et al., 2014; Parker et al., 2014; Tanev et al., 2014), shift-workers (Lo et al., 2010), insomnia (Jiang et al., 2015; Maes et al., 2014), psychosomatic disorders (Salvioli et al., 2015) and major mental disorders such as schizophrenia, anxiety and mood disorders (Akar et al., 2014; Clamor et al., 2014; Kemp et al., 2014; Moon et al., 2013; Sanchez-Gonzalez et al., 2014; Valenza et al., 2013). Particularly, as far as mood disorders are concerned, changes in the features derived from HRV analysis were found in Major Depression (Stapelberg et al., 2012; Wang et al., 2013) and, recently, in Bipolar Disorder patients, who, as a group, showed a significantly decreased HRV and parasympathetic activity as compared to healthy individuals (Cohen et al., 2003; Henry et al., 2010). Finally, a recent study highlighted that the steeper the reduction of HRV, the more severe was the clinical symptomatology both in bipolar and schizophrenic patients (Quintana et al., 2015).

On the other hand, modulation of the autonomic nervous system seems to have relevant effects on the central nervous system both under physiological, and even more so, pathological conditions. For instance, vagal nerve stimulation (VNS) has been widely applied in patients with Major Depression so to become approved by FDA for the treatment of drug-resistant depression (Berry et al., 2013; Martin and Martín-Sánchez, 2012). However, results on VNS efficacy remain inconsistent and largely based on open-labeled studies (Daban et al., 2008). Another indication of the potential link between autonomic nervous system and mood is provided by the use of relaxation techniques based on autonomic nervous system modulation, including cardio-feedback. These techniques are widely used for managing stress and negative emotions (Karavidas et al., 2007; Lehrer and Gevirtz, 2014). Nonetheless, the pathophysiological relationship between ANS and mood is still debated (Thayer and Lane, 2009). Recently, it has been suggested that the relationship between HRV and mood may be based on the role of parasympathetic activity, which affects the cardiac dynamics and plays a role in emotional regulation and flexibility, as well as in social cognition (Chang et al., 2013; Cristea et al., 2014). For instance, recent studies have shown an association between HRV and cerebral blood flow in the middle prefrontal cortex, which is actively engaged during emotional modulation tasks (Chang et al., 2013; Thayer et al., 2012). On the basis of these results, the authors claimed that HRV may be an index of the activity of a central autonomic network that directly influences both the heart and the control over behavior (Thayer et al., 2012). Moreover, HRV alterations were thought to be in a strict relationship with cholinergic mediated inflammatory modulation and with changes in endocrine and allostatic systems (Alamili et al., 2015; Kenney and Ganta, 2014). It is interesting 
to note that such alterations also may play a role in the pathophysiology of mood disorders (Kemp and Quintana, 2013).

Therefore, HRV metrics seem to reflect clinical states in mood disorders, although they lack specificity, as HRV alterations are shared among mental disorders and several somatic conditions.

In light of these considerations, within the framework of the European project PSYCHE (Personalized monitoring SYstems for Care in mental HEalth), we have developed the PSYCHE platform for the longitudinal physiological monitoring of Bipolar Disorder patients through comfortable wearable systems (Javelot et al., 2014). The PSYCHE system was not primarily conceived to unveil the pathophysiology of ANS alterations in mood disorders, but mainly to provide indices that may be useful for the clinical management of bipolar patients. The PSYCHE platform consisted of a wearable sensorized tshirt, embedded with fabric-based electrodes that acquire ECG, respiration signals, and body activity, as well as a smartphone, collecting data from the wearable system via Bluetooth technology and that could be used to record voice parameters and subjective data (e.g., mood and sleep agendas) (Valenza et al., 2013, 2014b). All the data from each individual patient, including both the parameters acquired from the system and the clinical and medication data, were stored in a webportal (Javelot et al., 2014).

We previously demonstrated that this system can lead to an automatic classification of mood states in bipolar disorder patients, which is highly consistent with the clinical diagnosis (Valenza et al., 2014a, 2015a, 2013, 2014b). It should be underlined that such a highly accurate classification was obtained using a multi-feature analysis, that is, by employing information derived from multiple HRV features to classify the mood states, and not only from a single one, contrasting most previous studies (e.g., (Chang et al., 2014; Cohen et al., 2003; Henry et al., 2010; Latalova et al., 2010)). This aspect is particularly relevant because the use of multiple features in classification algorithms may help overcoming the above-mentioned specificity issue of HRV metrics.

Moreover, all the studies published to date measured HRV only once, and therefore did not provide any longitudinal evaluation. On the contrary, in our previous studies, we described a potentially useful tool for the longitudinal clinical management of mood disorder patients. HRV analysis may provide a very precise estimation of the mood state of a patient remotely and in the absence of any direct clinical evaluation (Valenza et al., 2013). Therefore, the PSYCHE system should be regarded as a clinical decision support tool and not as an alternative to the clinician assessment. Indeed, HRV monitoring does not replace the clinical interview (which remains the gold standard for mood evaluation), but can rather provide an accurate estimation that may contribute to monitor the patient state in the periods between specialistic evaluations. This estimation is highly consistent with the clinical interview, and becomes more accurate when data from previous mood state evaluations are considered (Valenza et al., 2014a, 2014b).

The above-mentioned observations prompted us to hypothesize that the current mood state may contain some psychophysiological features that will be expressed in the following one. If this is the case, then we expect that mood automatic classification will be improved if information from subsequent mood states is taken into account. Specifically, we hypothesized that mood swings occurring during the clinical course of Bipolar Disorder possess physiological features, related to cardiovascular dynamics, that depend on the previous mood state and that contain information related to the subsequent state as well. In this view, Bipolar Disorder should be regarded in its whole longitudinal course, considering the symptoms transition in a 'continuum' and not just as a series of states that simply alternate without any relationship among each other. This is in line also with clinical observations: for instance, a cycle in which mania follows depression and precedes euthymia is associated with a longer depressive status and a lesser intense response to mood stabilizers as compared to a cycle in which depression follows mania and precedes euthymia
(Koukopoulos et al., 2013).

To test this hypothesis, we conducted a study in a subgroup of Bipolar Disorder patients to assess whether the HRV-based classification of mood states is affected by the clinical course of the illness, that is, by the mood states that precede and follow a target state. In other words, the recognition, for instance, of a mixed state through its HRV feature representation (HRV-fr) at time T would be improved by taking into account the mood state - that is depression, mixed, mania or euthymia - at time $\mathrm{T}-1$ and $\mathrm{T}+1$, respectively.

\section{Methods}

\subsection{Patients with $B D$}

We studied long-term HRV dynamics in eight Bipolar Disorder patients $(\mathrm{F} / \mathrm{M}=3 / 5$; mean age \pm s.d. $=40 \pm 10$ years $)$. All the patients signed a written informed consent document before the enrollment into the study. The study, conducted under the PSYCHE protocol, was approved by the local Ethical Committees at the participating institutions, namely, Pisa University Hospital and University of Strasburg. Six patients had Bipolar I disorder and two had Bipolar II disorder according to the DSM-IV-TR criteria (APA, 2000). Diagnoses were performed by board-certified psychiatrists or clinical psychologists. Patients were recruited at the Pisa University Hospital and at the Strasbourg University Hospital. All the patients underwent a complete medical interview during which a physician reviewed their medical charts to exclude presence or history of any cardiac or somatic disease that may affect heart dynamics. The two patients recruited in Pisa were inpatients at the psychiatric ward while the six patients in Strasbourg were outpatients. All patients were suffering from an active mood episode at the moment of recruitment and were recorded until remission was achieved and (for the two inpatients) they were discharged from the hospital ward (Table 1). Fig. 1 illustrates the timeline of the study for each patient.

The clinical labeling of the mood states was performed according to the Young Mania Rating Scale (YMRS) (Young et al., 1978) and the Quick Inventory of Depressive Symptomatology (QIDS-C) (Rush et al., 2003) scores. YMRS is an 11 items rating scale to assess maniac symptoms and it is based on the patient's subjective report of his or her clinical condition and on clinical observations made during the course of the psychiatric interview. The QIDS-C is a 16 items scale used to assess depressive symptoms based on the patient's report.

For our purpose we considered a YMRS score greater than six as indicating a hypomaniac state; a QIDS-C higher than eight as indicating a depressive state; mixed state was considered if both the scales were over the above-mentioned cuts-off. Both YMRS and QIDS-C were administered by a clinical psychologist or a board-certified psychiatrist

Table 1

Patients demographic and clinical information, including site of recruitment, age, gender, therapy (drug classes) and clinical evaluation at each acquisition.

\begin{tabular}{|c|c|c|c|c|c|c|c|c|c|}
\hline \multicolumn{4}{|c|}{ Demographics } & \multicolumn{6}{|c|}{ Acquistion } \\
\hline ID & gender & age & treatment & 1 & 2 & 3 & 4 & 5 & 6 \\
\hline Stras1 & M & 39 & BDZ. MDS. AD. AP & HY & HY & $\mathrm{HY}$ & ES & ES & \\
\hline Stras2 & M & 51 & BDZ. MDS AD. AP & HY & ES & $\mathrm{HY}$ & $\mathrm{HY}$ & & \\
\hline Stras3 & M & 39 & MDS, AD. AP & HY & $\mathrm{HY}$ & ES & ES & ES & \\
\hline Stras4 & M & 33 & $\mathrm{MDS}, \mathrm{AD}, \mathrm{AP}$ & DP & DP & DP & DP & DP & ES \\
\hline Stras5 & $\mathrm{F}$ & 54 & $\mathrm{MDS}, \mathrm{AD}, \mathrm{AP}$ & DP & DP & $\mathrm{HY}$ & DP & ES & \\
\hline Stras6 & $\mathrm{F}$ & 37 & MDS. AD & HY & HY & ES & ES & ES & \\
\hline Pisa1 & $\mathrm{F}$ & 32 & BDZ. MDS. AD. AP & DP & DP & ES & & & \\
\hline Pisa2 & M & 56 & MDS. AD & MS & MS & DP & DP & DP & ES \\
\hline mean & & 40.5 & & & & & & & \\
\hline st.dev & & 9.7 & & & & & & & \\
\hline
\end{tabular}

BDZ: benzodiazepine and/or zolpidem; MDS: mood stabilizers; AP: antipsychotics; AD: antidepressants; HY: Hypomania; DP: Depression; ES: Eutymic state; MS: mixed state. 


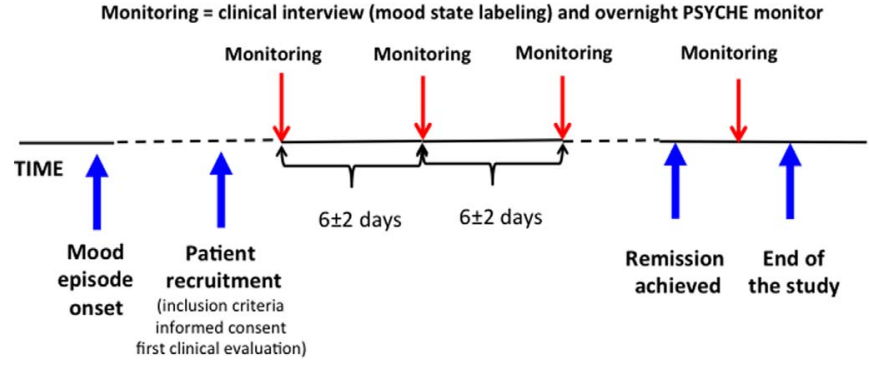

Fig. 1. Schematic time-line of the study. Patients suffering by an active mood episode were recruited for the study. Patients were studied a minimum of three times and a maximum of six times. The mean (standard deviation) interval between two contiguous registrations was $6( \pm 2)$ days.

trained for the administrations of the two scales and after reaching an acceptable inter-rate reliability. The clinical labeling was done the same day of the HRV evaluation performed with the PSYCHE system.

Because of the observational nature of this study, patients were taking different combinations of antidepressant, mood stabilizer and/ or antipsychotic drugs (Table 1).

\subsection{Data acquisition}

We collected 39 ECG recordings (11 hypomaniac, 13 depressive, 2 mixed and 13 euthymic states) (Table 1) with a sampling frequency of $256 \mathrm{~Hz}$. ECG series were processed and analyzed using a self-coded Matlab software and related toolboxes for digital signal processing (see Supplementary Materials for further details).

Overall, each patient was followed for a minimum of three and a maximum of six recording sessions, for a total of more than $300 \mathrm{~h}$ of recordings. The mean ( \pm standard deviation) interval between each recording was $6 \pm 2$ days. The mean ( \pm standard deviation) length of the recordings was $16 \pm 1 \mathrm{~h}$. Table 2 shows the mean ( \pm standard deviation) of the scores of QIDS and YMRS for each state.

\subsection{The PSYCHE platform system}

To collect psychophysiological data we used the core sensing system of the PSYCHE project, i.e., the wearable monitoring platform. The system was developed by Smartex s.r.l. (Pisa, Italy) and consists of a comfortable sensorized $\mathrm{t}$-shirt with dry textile-based electrodes that acquire the patient's ECG, a piezo-resistive sensor to acquire the respiration signal, and a three-axial accelerometer. The system is easy to use and reaches electrochemical equilibrium between the skin and electrodes after just a few minutes, allowing for a high and stable quality of the signal. While using the Psyche system, patients were instructed to freely perform their usual daily-life activities either at home or in the hospital ward, while the afore-mentioned physiological signals were monitored and stored in a microSD card. A smartphone received through a Bluetooth connection the data from the wearable system and sent it to a remote server wherein the processing algorithms were located. Further information on the Psyche project can be found in (Javelot et al., 2014; Valenza et al., 2013).

Table 2

Scores for the Young Mania Rating Scale (YMRS) and the Quick Inventory of Depressive Symptomatology (QIDS-C) for each type of mood state (mean \pm st.dev).

\begin{tabular}{llllll}
\hline & \multicolumn{2}{l}{ QIDS } & & & \multicolumn{2}{l}{ YMRS } \\
\cline { 2 - 3 } \cline { 5 - 6 } & Mean & St.dev & & Mean & St.dev \\
\hline mixed state & 12.00 & 3.00 & & 10.50 & 1.4 \\
hipomaniac state & 4.56 & 2.36 & & 9.18 & 2.13 \\
depressive state & 12.05 & 3.39 & & 1,00 & 2.33 \\
euthimic state & 2.26 & 2.08 & & 2,00 & 2.29 \\
\hline
\end{tabular}

\subsection{Data analysis}

For this study we considered only data extracted from the ECG signal. Specifically, we considered HRV features defined in a) the time domain: mean (HRVmean), standard deviation (HRVstd), square root of the mean of the sum of the squares of differences between adjacent inter-beats (RMSSD), normalized number of pairs of adjacent interbeats differing by more than $50 \mathrm{~ms}$ (pNN50); b) the frequency domain: spectral power in the VLF, LF, HF and their ratio (LF/HF): c) entropic domains: approximate entropy (ApEn) and sample entropy (SampEn) and detrended fluctuation indices (DFA- $\alpha_{1}, \alpha_{2}$ ) (Valenza et al., 2013, 2014b). The features extraction procedure was performed on continuous, artifact-free, five-minute long recordings of heartbeat dynamics producing a Heart Rate Variability feature representation (HRV-fr) 'example'. Parts of the signals with artifacts and artifact-free ECG signals lasting less than 5 min were discarded and not considered for further analysis. The length of five minutes was chosen in agreement with the current guidelines and in order to fulfill the signal processing requirements of stationarity for long-term HRV series (Rajendra Acharya et al., 2006; Task Force of the European Society of Cardiology, 1996). A more detailed description of the algorithms used for HRV extraction, artifact correction and HRV-features calculation can be found in the Supplementary Materials.

Each 'example' was labeled according to the mood status defined by the clinical evaluation described above. Every mood state of a given Bipolar Disorder patient is therefore represented as a set of 'examples'. Support vector machines (SVM) algorithms with nonlinear radial basis kernel were used to test the accuracy of the automatic labeling of the 'examples'. In order to test whether a relationship was present among subsequent mood states, we trained and compared results coming from four SVM algorithms, which take as input four different HRV-fr sets:

1) The HRV-fr set belonging to each mood state was considered alone and as independent from data coming from other mood states.

2) The HRV-fr set belonging to each mood state was normalized using the information coming from the previous mood state in time.

3) The HRV-fr set belonging to each mood state was normalized using the information coming from the previous and subsequent mood states.

4) The HRV-fr set belonging to each mood state was normalized using the information coming from two randomly chosen mood states to control for differences in accuracy exclusively due to a double normalization.

Details including the mathematical formulation of the normalization procedure are reported in the Supplementary Materials. Therefore, the first acquisition of each subject provided HRV-fr sets for procedures 1) and 4); the last acquisition for each subject provided HRV-fr sets for the procedures 1), 2) and 4) and all the other acquisitions provided HRV-fr sets for all the described procedures. Since the subject Pisa1 had only three acquisitions, we could not compute the HRV-fr set with procedure 4).

The SVM classification algorithms were run for each patient individually, thus implementing a personalized intra-subject analysis.

Differences in classification accuracy (i.e., the average of sensibility and specificity) among all the patients, achieved from the four kinds of HRV-fr set, were tested through the non-parametric Friedman test for paired data.

\section{Results}

No physiological artifacts comprising ectopic beats and arrhythmic events were found in any of the analyzed HRV series. Comparing the four HRV-fr sets, we found that the normalization using information from previous and subsequent mood states produced a significantly higher average classification accuracy as compared to the other 
Table 3

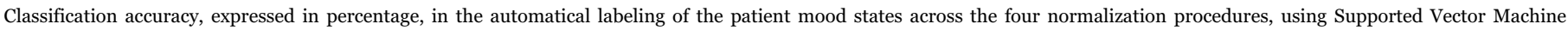
algorithms. Values are reported as median \pm inter-quartile range.

\begin{tabular}{|c|c|c|c|c|c|c|c|c|c|c|c|c|}
\hline \multirow[t]{2}{*}{ ID } & \multicolumn{3}{|c|}{ Independent characterization } & \multicolumn{3}{|c|}{$\begin{array}{l}\text { Mood depends on the previous } \\
\text { state }\end{array}$} & \multicolumn{3}{|c|}{$\begin{array}{l}\text { Mood depends on a random } \\
\text { state }\end{array}$} & \multicolumn{3}{|c|}{$\begin{array}{l}\text { Mood depends on the previous and } \\
\text { future states }\end{array}$} \\
\hline & Sens. & Spec. & Accuracy & Sens. & Spec. & Accuracy & Sens. & Spec. & Accuracy & Sens. & Spec. & Accuracy \\
\hline Stras1 & 91.99 & 19.6 & 55.79 & 88,64 & 70,8 & 79.72 & 96,11 & 51,68 & 73.89 & 99,99 & 98,47 & 99.23 \\
\hline Stras2 & 97.03 & 37.5 & 67.26 & 86,46 & 96,25 & 91.35 & 97,84 & 38,54 & 68.19 & 98,96 & 99,99 & 99.47 \\
\hline Stras3 & 88.33 & 56.02 & 72.18 & 88,59 & 95,18 & 91.89 & 87,11 & 76,77 & 81.94 & 100 & 100 & 100 \\
\hline Stras4 & 98.53 & 16.09 & 57.31 & 99,68 & 91,95 & 95.81 & 99,13 & 65,51 & 82.32 & & & \\
\hline Stras5 & 93.04 & 51.08 & 70.98 & 90,58 & 92,09 & 91.33 & 97,15 & 81,3 & 89.22 & 100 & 100 & 100 \\
\hline Stras6 & 68.92 & 86.02 & 77.47 & 10,81 & 97,13 & 53.97 & 73,65 & 94,62 & 84.14 & 94,59 & 100 & 97.30 \\
\hline Pisa1 & 96.34 & 54.44 & 75.39 & 97,47 & 86,67 & 92.07 & & & & & & \\
\hline Pisa2 & 65.86 & 78.52 & 72.19 & 78,08 & 93,9 & 85.99 & 87,26 & 64,38 & 75.82 & 99,32 & 99,73 & 99.52 \\
\hline mean & 87.51 & 49.91 & 68.57 & 80.04 & 90.5 & 85.26 & 91.83 & 68.07 & 79.36 & 98.81 & 99.7 & 99.25 \\
\hline st.dev & 12.85 & 25.07 & 8.01 & 28.75 & 8.6 & 13.55 & 9.19 & 18.77 & 7.1 & 2.11 & 0.61 & 1,00 \\
\hline
\end{tabular}

Sens.: sensibility; Spec.: specificity.

normalization procedures and to the independent characterization.

Accuracy was (mean \pm s.d.) $68.57 \pm 8.01$ for independent classification, $85.26 \pm 13.55$ for the normalization with the previous mood state, $79.30 \pm 7.10$ for the normalization with random mood states (not preceding nor following) and $99.25 \pm 1.00$ for the normalization with both previous and subsequent mood states. Friedman test showed a significant difference for the normalization with previous and subsequent mood states as compared to the others $(\mathrm{p}<0.001)$ (Table 3 ).

Box-plot statistics of all SVM-derived classification accuracies are shown in Fig. 2.

\section{Discussion}

We employed a novel integrated system - the PSYCHE platform that allows for a longitudinal, non-invasive recording of multiple physiological parameters to study heartbeat dynamics in BD patients over time. Our results indicate that the measure of HRV features accurately reflects the clinical labeling of mood states. Specifically, our findings show that labeling accuracy increases significantly when information from previous and subsequent mood states is included into the analysis. Altogether, our data provides the first experimental evidence that psychophysiological states in Bipolar Disorder patients influence each other: the preceding mood state has an influence on the current one, which, in turn, will influence and contains psychophysio- logical features of the following one.

Importantly, since data were analyzed at a single subject level, our results have two main clinical implications. First, the analysis of longitudinal HRV dynamics is an effective tool to monitor the clinical state of individual bipolar patients. Thus, the remote monitoring may provide clinicians with a valuable and reliable measure in the management of Bipolar Disorder. This is in line with previous results obtained by using the PSYCHE platform (Valenza et al., 2015a, 2013).

The second major implication of our results is related to the fact that the classification of mood states is significantly improved when considering measures from both the preceding and the subsequent psychophysiological states. Such an effect indicates that some aspects of the HRV-fr of the future state $(\mathrm{T}+1)$ may be already present in the target state (T0). If confirmed in larger samples of patients, this finding may be used to forecast the clinical course of the disorder in individual patients.

As compared to the previously published studies on HRV and mood disorders, our approach was multi-parametric, meaning it included several HRV features at the same time - to train an SVM classification algorithm - rather than considering only one (or a few). In contrast, the overwhelming majority of previous studies of this kind focused on specific alterations of only one, or at most a few, HRV parameters in patients as a group. Therefore, our results could also to be read within a wider framework. More specifically, psychiatry and clinical psychology

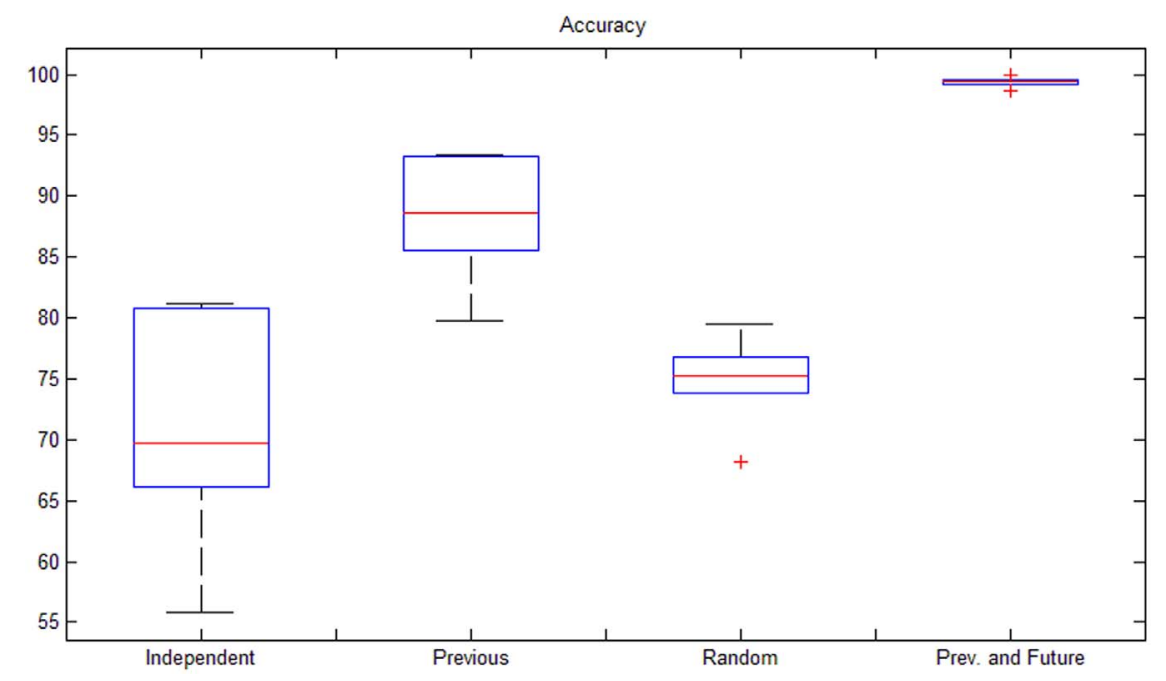

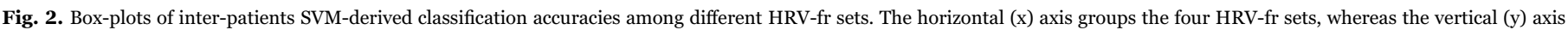

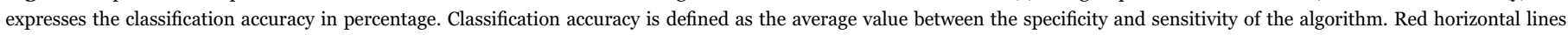

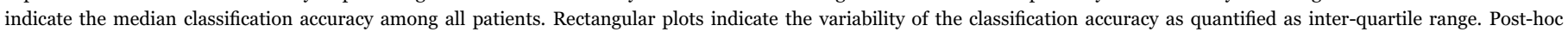
analysis performed after a Friedman test showed a significant difference for the latter normalization as compared to the others ( $<<0.001$ ). 
have suffered since their dawn from the lack of objective external indicators to confirm a diagnostic hypothesis or to monitor the clinical course of mental disorders. For years, an equivalent of what the glycemic index is for diabetes or what hemoglobin concentration measure is for anemias, has been searched without success. The possibility to use a multi-parametric analysis including several features that provide different information may be a new promising area in the study of specific markers for mood disorders. In this sense, it is relevant to underline that we used a multi-feature analysis for our classification, thus we did not rely on differences in just a single feature. Single feature analysis has both sensitivity and specificity problems (Valenza et al., 2013). Similar alterations in single HRV features are commonly found across several somatic and psychiatric conditions. A recent meta-analysis has considered different HRV features measured in psychiatric patients. The meta-analysis showed a consistent reduction of HRV features in patients as compared to controls (Alvares et al., 2016). However, the meta-analytic approach pooling the effect size of each study cannot highlight changes in individual features that may be specific for a given mental disorder. In turn, multi-feature analysis not only increases sensitivity but also specificity, as indicated also by the fact that we were able to classify different mood states with a higher accuracy.

Moreover, as mental disorders are not homogenous entities, one index (or the linear combination of a few indices) may not be sufficient to catch their intrinsic heterogeneity. Our work shows how a machine learning algorithm (i.e., the SVM algorithm) trained on several features can be a reliable tool for mood state labeling at a single subject level. Also, this tool reflects some neurobiological features of Bipolar Disorders, suggesting the necessity to consider bipolar cycles in their longitudinal course and not just as a series of distinct episodes of mood symptoms and states.

In the present study, we focused on the HRV dynamics, which several studies found altered in mood disorders patients at a group level. Patients suffering from Major Depression showed a reduction in HRV parameters, including HF power, when compared to both euthymic patients and healthy volunteers (Kemp et al., 2010; Liang et al., 2015; Wang et al., 2013). However, results were not completely consistent among studies. Kemp and colleagues (Kemp et al., 2014) found differences in depressed patients with melancholia as compared to healthy controls in resting-state HRV (including RMSSD, HF), but these differences were not significant when non-melancholic depressed patients were considered (Kemp et al., 2014).

Results comparing patients during a depressive episode to remitted ones are even more controversial. A recent work suggested that HRV alterations should be considered a trait marker of depression, since RMSSD and HF were found significantly lower not only in patients during a depressive episode, but also during remission (Brunoni et al., 2013). However, some authors claimed that an increase toward HRV normalization was linked to remission. For instance, successful antidepressant treatment with either fluoxetine or doxepine was found to be associated with HRV increases (Khaykin et al., 1998). Finally, the cardiac biofeedback seemed both to increase HRV and to reduce symptoms in patients with primary Major Depression (Karavidas et al., 2007) and in patients with coronary heart disease experiencing depressive symptoms (Patron et al., 2012). It is also relevant to underline that the different studies often examined different HRV measures. Consequently, a closer look at the results of the individual studies highlighted some inconsistencies. For instance, Moon and colleagues (Moon et al., 2013) found differences in HF in depressed patients as compared to healthy controls, but failed to find differences in total power and in HF/LF as revealed instead by other studies (Brunoni et al., 2013; Liang et al., 2015; Wang et al., 2013).

The literature on Bipolar Disorders is even less consistent, while conversely more limited. Again, typically reduced HRV has been linked to a manic clinical state (Chang et al., 2014; Cohen et al., 2003; Henry et al., 2010; Latalova et al., 2010; Moon et al., 2013). Moreover, a decreased HRV was associated with increased severity in both mood disorders and in schizophrenia (Chang et al., 2014; Quintana et al., 2015). As for Major Depression, HRV alterations seem to be a trait marker for Bipolar Disorder since they were present also during the euthymic state (Cohen et al., 2003; Latalova et al., 2010), though other studies failed to identify HRV alterations in euthymic bipolar subjects as compared to control healthy individuals (Henry et al., 2010; Todder et al., 2005). Finally, we recently showed that complexity measures of the HRV may correlate with the clinical status of the patient and can be used as an objective measure of the course of the disease (Lanata et al., 2015). However, as in Major Depression, individual studies of Bipolar Disorders highlighted a reduction of some HRV metrics that differed from study to study (Chang et al., 2014; Cohen et al., 2003; Henry et al., 2010; Latalova et al., 2010; Moon et al., 2013). For instance, recent work showed that un-medicated bipolar patients in maniac phase exhibited significantly lower HRV mean, HRV variance, LF, and HF and higher LF/HF compared to controls (Chang et al., 2014). Other studies partially failed to replicate these differences and found, in contrast, other significant alterations (Henry et al., 2010; Moon et al., 2013).

We claim that a part of this inconsistency may be related to the approach used for data analysis. While most of the studies used one or a few HRV metrics to characterize their sample and to make comparisons at a group level, a single-variable approach is not robust enough to characterize mood swings and states in Bipolar Disorders when looking at the individual patient (Valenza et al., 2013). This may also be related to the neurobiological and psychophysiological heterogeneity of Mood Disorders. Although a dysfunction of the autonomic central network has been suggested as a potential correlate of mood disorders (Kemp and Quintana, 2013), the entity of such an alteration likely may vary among patients. In a previous pilot work, we defined an effective pattern of HRV-fr able to discern different pathological mental states in Bipolar Disorder patients at a single level (Valenza et al., 2013, 2014b). More recently, we observed that the onset of a new mood state brings the past clinical history along, and the identification of the next state is more accurate if the previous one is considered (Valenza et al., 2014b). Therefore, a better understanding of the patient's mood status can be achieved considering the dynamics of the disorder rather than the single observation treated as completely independent.

In the present study, we extended these results by demonstrating that the classification accuracy significantly increases when HRV-fr classification takes into account information from previous and subsequent mood states. Thus, while the clinical state may change completely from a state to the next one, some psychophysiological fingerprints remain from the previous state. In the same way, some features may already be changed and be already related to the subsequent mood state while, at a clinical level, such change is not yet detectable.

\subsection{Study limitations and their relationship with the significance of the study}

The present study highlights several previously unknown characteristics of the cardiovascular dynamics in Bipolar Disorder patients. Importantly, our results are significant at a single subject level and are highly consistent across patients, despite individual differences in clinical features and treatment. However, there are a few limitations that warrant to consider these findings as preliminary.

The first, and probably the most important one, is the sample size. Given the high heterogeneity of Bipolar Disorder patients, to sustain a generalization of our results that fits all the different clinical and psychophysiological presentations of this disorder could turn out to be rather speculative. Even if having more patients may be irrelevant in terms of statistical significance, since we performed a single subject analysis, it would strengthen our approach by replicating its feasibility across a wide spectrum of Bipolar Patients. 
Similar considerations can be drawn for treatment. Although the literature is not completely consistent, several pieces of evidence indicate that HRV may be affected by medications, specifically, by antipsychotic and tricyclic drugs (Alvares et al., 2016; Kemp et al., 2010, 2014; Udupa et al., 2007). The Psyche project was born as an observational study to evaluate the psychophysiological correlates of the clinical course of Bipolar Disorder and, therefore, no manipulation of the treatment prescribed by the patient psychiatrists was allowed. The validation of the Psyche wearable system for studying longitudinal dynamics in Bipolar Disorder patients may encourage additional studies in larger patient samples, including never medicated, first episode patients who may be assigned to a specific treatment with a given drug (or class of drugs). This strategy would contribute to generalize our results and to disentangle the potential effects of different medications on HRV classification.

Another potential limitation that requires future assessments in larger samples, is the fact that we cannot completely rule out that heartbeat dynamics may have been affected also by lifestyles, circadian rhythms or by the presence of other pathological conditions a part form those considered as exclusion criteria. The experimental design of our study, however, was such to minimize any of the above potential confounds. Specifically, we did not include patients with any somatic disease, and in particular cardiovascular or neural disorders, which may affect heartbeat dynamics, as well as HRV features. Moreover, our experimental setup foresaw long-term (up to $16 \mathrm{~h}$ ) recordings, from late afternoon to the next morning. Therefore, most of the recording sessions covered a period in which the patients slept or were in bed. This should minimize the effects of different lifestyles and times of the day on HRV. In addition and more importantly, the analysis was performed at a single subject level, so that data cannot be affected by differences in habits and lifestyles, as they presumably remained constant in each individual subject.

Finally, the use of a multi-parametric approach has a potential drawback: our analysis did not allow us to identify the contribution of individual features. Therefore, in the present study, the physiological meaning remains partially undefined. A deeper understanding of the HRV alterations that are related to clinical changes in bipolar disorder would greatly contribute to clarify the pathophysiology of autonomic nervous system activity in Mood Disorders. For instance, it would be helpful to evaluate whether HRV alterations in mood disorders are related to alterations of the emotional modulation process (related to abnormal activity in the middle prefrontal cortex) or to the cholinergic mediated alterations of the inflammatory mechanisms (Kemp and Quintana, 2013). Futhermore, understanding which are the most relevant features and how they change along with the changing in mood state would foster the development of algorithms to forecast clinical course in Bipolar Disorders. As a matter of fact, in another paper based on data gathered from the PSYCHE project, we began to explore this issue (Valenza et al., 2016). Briefly, we were able to predict whether each patient would be in an active state of illness or in remission at the subsequent observation. We were also able to conclude that the most informative features for mood state prediction in bipolar disorder were: the standard deviation of the RR intervals (StdRR); Square root of the mean squared difference between adjacent R-R (RMSSD); Poincare SD1 and SD2 and HF power, which are all related to parasympathetic activity. Therefore, even if the analyses reported in this study are different than the ones in the Valenza et al. (2016) paper, our findings suggest that HRV features related to vagal activity are those that are mainly related to mood changes and mood changes prediction. However, further studies involving a larger number of patients are needed to address this issue, by studying the role and weight of each single HRV metrics. Also, we acknowledge the fact that during the routine clinical visits, specialists do not have information on future clinical states and therefore the present results cannot have a direct impact on clinical practice as it is. Indeed, the presence of future mood state characteristics in the actual mood state allows us to speculate that this information may be used to predict the clinical outcome on the basis of the HRV characteristics. Furthermore, following the outcomes of the present study, we developed a decision support system, which uses information contained in the current mood state to predict future outcome (preliminary results were described in (Valenza et al., 2015b)).

\subsection{Conclusions}

The results of the present study provide for the first time a psychophysiological evidence, though preliminary and in need of replication, that mood states should be considered as a longitudinal sequence, since each HRV-fr in a given state is related to those from the preceding and subsequent mood states. Importantly, such characteristics were identified at a single subject level and therefore may be implemented to monitor the course of mood episodes in Bipolar Disorder and to develop algorithm that may contribute to predict clinical course. Our findings provide additional support to the role of central autonomic network alterations in the pathophysiology of mood disorders. We believe that a constant monitoring and feedback to both patients and physicians are not only new keys to managing mental illness and, ultimately, to identify and forecast critical clinical events, but also an innovative approach to examine the dysfunction within the central autonomic network.

Finally, we consider the multi-parametric approach to HRV and, more in general, to biological signals in course of psychiatric disorders, a promising approach toward the definition of much-needed objective parameters in psychopathology.

\section{Acknowledgements}

The study was supported by and conducted within the PSYCHE Project, granted by EU Commission (FP7 ICT-247777 Psyche).

The study was approved by the local ethical committees at the Pisa University-Hospital (Comitato Etico di Area Vasta Nord Ovest Comitato Etico Sperimentazione Farmaco - CESF), Pisa, Italy and at the Strasbourg University Hospital (Comité de Protection des Personnes "Est IV").

\section{Appendix A. Supplementary material}

Supplementary data associated with this article can be found in the online version at doi:10.1016/j.jad.2016.11.008.

\section{References}

Adlan, A.M., Lip, G.Y.H., Paton, J.F.R., Kitas, G.D., Fisher, J.P., 2014. Autonomic function and rheumatoid arthritis-A systematic review. Semin. Arthritis Rheum. 44, 283-304.

Ahmed, M.W., Kadish, A.H., Parker, M.A., Goldberger, J.J., 1994. Effect of physiologic and pharmacologic adrenergic stimulation on heart rate variability. J. Am. Coll. Cardiol. 24, 1082-1090.

Akar, S.A., Kara, S., Latifoğlu, F., Bilgiç, V., 2014. Analysis of heart rate variability during auditory stimulation periods in patients with schizophrenia. J. Clin. Monit. Comput..

Alamili, M., Rosenberg, J., Gögenur, I., 2015. Day-night variation in heart rate variability changes induced by endotoxaemia in healthy volunteers. Acta Anaesthesiol. Scand. $59,457-464$.

Alvares, G.A., Quintana, D.S., Hickie, I.B., Guastella, A.J., 2016. Autonomic nervous system dysfunction in psychiatric disorders and the impact of psychotropic medications: a systematic review and meta-analysis. J. Psychiatry Neurosci. 41, 89-104.

Antonijevic, I., 2008. HPA axis and sleep: identifying subtypes of major depression. Stress 11, 15-27.

APA, 2000. Diagnostic and statistical manual of mental disorders: dsm-iv-tr. American Psychiatric Pub., Arlington, VA.

Arnone, D., McIntosh, A.M., Ebmeier, K.P., Munafò, M.R., Anderson, I.M., 2012. Magnetic resonance imaging studies in unipolar depression: systematic review and meta-regression analyses. Eur. Neuropsychopharmacol. 22, 1-16.

Aubert, A.E., Vandeput, S., Beckers, F., Liu, J., Verheyden, B., Van Huffel, S., 2009 Complexity of cardiovascular regulation in small animals. Philos Trans. A Math. Phys. Eng. Sci. 367, 1239-1250. 
Berry, S.M., Broglio, K., Bunker, M., Jayewardene, A., Olin, B., Rush, A.J., 2013. A patient-level meta-analysis of studies evaluating vagus nerve stimulation therapy for treatment-resistant depression. Med. Devices (Auckl.) 6, 17-35.

Brunoni, A.R., Kemp, A.H., Dantas, E.M., Goulart, A.C., Nunes, M.A., Boggio, P.S., Mill, J.G., Lotufo, P.A., Fregni, F., Benseñor, I.M., 2013. Heart rate variability is a trait marker of major depressive disorder: evidence from the sertraline vs. electric current therapy to treat depression clinical study. Int. J. Neuropsychopharmacol. 16, 1937-1949.

Chang, C., Metzger, C.D., Glover, G.H., Duyn, J.H., Heinze, H.-J., Walter, M., 2013. Association between heart rate variability and fluctuations in resting-state functional connectivity. Neuroimage 68, 93-104.

Chang, H.-A., Chang, C.-C., Tzeng, N.-S., Kuo, T.B.J., Lu, R.-B., Huang, S.-Y., 2014 Heart rate variability in unmedicated patients with bipolar disorder in the manic phase. Psychiatry Clin. Neurosci. 68, 674-682.

Clamor, A., Hartmann, M.M., Köther, U., Otte, C., Moritz, S., Lincoln, T.M., 2014 Altered autonomic arousal in psychosis: an analysis of vulnerability and specificity. Schizophr. Res. 154, 73-78.

Cohen, H., Kaplan, Z., Kotler, M., Mittelman, I., Osher, Y., Bersudsky, Y., 2003. Impaired heart rate variability in euthymic bipolar patients. Bipolar Disord. 5, 138-143.

Cristea, I.A., Valenza, G., Scilingo, E.P., Szentágotai Tătar, A., Gentili, C., David, D., 2014. Autonomic effects of cognitive reappraisal and acceptance in social anxiety: evidence for common and distinct pathways for parasympathetic reactivity. J. Anxiety Disord. 28, 795-803.

Crosswell, A.D., Lockwood, K.G., Ganz, P.A., Bower, J.E., 2014. Low heart rate variability and cancer-related fatigue in breast cancer survivors. Psychoneuroendocrinology 45 , $58-66$.

Daban, C., Martinez-Aran, A., Cruz, N., Vieta, E., 2008. Safety and efficacy of Vagus nerve stimulation in treatment-resistant depression. A systematic review. J. Affect. Disord. $110,1-15$.

Feng, J., Wang, A., Gao, C., Zhang, J., Chen, Z., Hou, L., Luo, C., Jiang, Y., Pan, J., 2014. Altered heart rate variability depends on the characteristics of coronary lesions in stable angina pectoris. Anadolu Kardiyol. Derg.

Goldstein, D.S., Bentho, O., Park, M.Y., Sharabi, Y., 2011. Low-frequency power of heart rate variability is not a measure of cardiac sympathetic tone but may be a measure of modulation of cardiac autonomic outflows by baroreflexes. Exp. Physiol. 96 , $1255-1261$.

Gonçalves, T.R., Farinatti, P., Gurgel, J.L., Soares, P.Pd.S., 2014. Correlation between cardiac autonomic modulation in response to orthostatic stress and indicators of quality of life, physical capacity, and physical activity in healthy individuals. J. Strength Cond. Res..

Harris, P.R.E., Stein, P.K., Fung, G.L., Drew, B.J., 2014. Heart rate variability measured early in patients with evolving acute coronary syndrome and 1-year outcomes of rehospitalization and mortality. Vasc. Health Risk Manag. 10, 451-464.

Heathers, J.A.J., 2012. Sympathovagal balance from heart rate variability: an obituary. Exp. Physiol. 97, 556.

Henry, B.L., Minassian, A., Paulus, M.P., Geyer, M.A., Perry, W., 2010. Heart rate variability in bipolar mania and schizophrenia. J. Psychiatr. Res. 44, 168-176.

Holman, A.J., Ng, E., 2008. Heart rate variability predicts anti-tumor necrosis factor therapy response for inflammatory arthritis. Auton. Neurosci. 143, 58-67.

Javelot, H., Spadazzi, A., Weiner, L., Garcia, S., Gentili, C., Kosel, M., Bertschy, G., 2014. Telemonitoring with respect to mood disorders and information and communication technologies: overview and presentation of the PSYCHE project. BioMed Res. Int. 2014, 104658.

Jiang, W., 2008. Impacts of depression and emotional distress on cardiac disease. Clevel. Clin. J. Med. 75 (Suppl 2), S20-S25.

Jiang, X.-L., Zhang, Z.-G., Ye, C.-P., Lei, Y., Wu, L., Zhang, Y., Chen, Y.-Y., Xiao, Z.-J., 2015. Attenuated or absent HRV response to postural change in subjects with primary insomnia. Physiol. Behav. 140C, 127-131.

Jones, K.I., Amawi, F., Bhalla, A., Peacock, O., Williams, J.P., Lund, J.N., 2014. Assessing surgeon stress when operating using Heart rate variability and the state trait anxiety Inventory: will surgery be the death of us? Colorectal Dis..

Karavidas, M.K., Lehrer, P.M., Vaschillo, E., Vaschillo, B., Marin, H., Buyske, S., Malinovsky, I., Radvanski, D., Hassett, A., 2007. Preliminary results of an open label study of heart rate variability biofeedback for the treatment of major depression. Appl. Psychophysiol. Biofeedback 32, 19-30.

Kemp, A.H., Quintana, D.S., 2013. The relationship between mental and physical health: insights from the study of heart rate variability. Int. J. Psychophysiol. 89, 288-296.

Kemp, A.H., Quintana, D.S., Gray, M.A., Felmingham, K.L., Brown, K., Gatt, J.M., 2010. Impact of depression and antidepressant treatment on heart rate variability: a review and meta-analysis. Biol. Psychiatry 67, 1067-1074.

Kemp, A.H., Quintana, D.S., Quinn, C.R., Hopkinson, P., Harris, A.W.F., 2014. Major depressive disorder with melancholia displays robust alterations in resting state heart rate and its variability: implications for future morbidity and mortality. Front. Psychol. 5, 1387.

Kenney, M.J., Ganta, C.K., 2014. Autonomic nervous system and immune system interactions. Compr. Physiol. 4, 1177-1200.

Khaykin, Y., Dorian, P., Baker, B., Shapiro, C., Sandor, P., Mironov, D., Irvine, J. Newman, D., 1998. Autonomic correlates of antidepressant treatment using heartrate variability analysis. Can. J. Psychiatry 43, 183-186.

Koukopoulos, A., Reginaldi, D., Tondo, L., Visioli, C., Baldessarini, R.J., 2013. Course sequences in bipolar disorder: depressions preceding or following manias or hypomanias. J. Affect Disord. 151, 105-110.

Lanata, A., Valenza, G., Nardelli, M., Gentili, C., Scilingo, E.P., 2015. Complexity index from a personalized wearable monitoring system for assessing remission in menta health. IEEE J. Biomed. Health Inf. 19, 132-139.

Latalova, K., Prasko, J., Diveky, T., Grambal, A., Kamaradova, D., Velartova, H., Salinger,
J., Opavsky, J., 2010. Autonomic nervous system in euthymic patients with bipolar affective disorder. Neuro Endocrinol. Lett. 31, 829-836.

Lehrer, P.M., Gevirtz, R., 2014. Heart rate variability biofeedback: how and why does it work. Front. Psychol. 5, 756.

Leistedt, S.J.-J., Linkowski, P., Lanquart, J.-P., Mietus, J.E., Davis, R.B., Goldberger, A.L., Costa, M.D., 2011. Decreased neuroautonomic complexity in men during an acute major depressive episode: analysis of heart rate dynamics. Transl. Psychiatry 1 , e27.

Liang, C.-S., Lee, J.-F., Chen, C.-C., Chang, Y.-C., 2015. Reactive heart rate variability in male patients with first-episode major depressive disorder. Prog. Neuropsychopharmacol. Biol. Psychiatry 56, 52-57.

Lichtman, J.H., Froelicher, E.S., Blumenthal, J.A., Carney, R.M., Doering, L.V., FrasureSmith, N., Freedland, K.E., Jaffe, A.S., Leifheit-Limson, E.C., Sheps, D.S., Vaccarino, V., Wulsin, L., Epidemiology, A.H.A.S.CotCo, Prevention, Cardiovascular, tCo, Nursing, S., 2014. Depression as a risk factor for poor prognosis among patients with acute coronary syndrome: systematic review and recommendations: a scientific statement from the American Heart Association. Circulation 129, 1350-1369.

Liu, Y.-C., Hung, C.-S., Wu, Y.-W., Lee, Y.-C., Lin, Y.-H., Lin, C., Lo, M.-T., Chan, C.-C., Ma, H.-P., Ho, Y.-L., Chen, C.-H., 2013. Influence of non-alcoholic fatty liver disease on autonomic changes evaluated by the time domain, frequency domain, and symbolic dynamics of heart rate variability. PLoS One 8, e61803.

Lo, S.-H., Lin, L.-Y., Hwang, J.-S., Chang, Y.-Y., Liau, C.-S., Wang, J.-D., 2010. Working the night shift causes increased vascular stress and delayed recovery in young women. Chrono-. Int 27, 1454-1468.

Lotufo, P.A., Valiengo, L., Benseñor, I.M., Brunoni, A.R., 2012. A systematic review and meta-analysis of heart rate variability in epilepsy and antiepileptic drugs. Epilepsia $53,272-282$.

Maes, J., Verbraecken, J., Willemen, M., De Volder, I, van Gastel, A., Michiels, N., Verbeek, I., Vandekerckhove, M., Wuyts, J., Haex, B., Willemen, T., Exadaktylos, V., Bulckaert, A., Cluydts, R., 2014. Sleep misperception, EEG characteristics and Autonomic Nervous System activity in primary insomnia: a retrospective study on polysomnographic data. Int. J. Psychophysiol. 91, 163-171.

Martin, J.L.R., Martín-Sánchez, E., 2012. Systematic review and meta-analysis of vagus nerve stimulation in the treatment of depression: variable results based on study designs. Eur. Psychiatry 27, 147-155.

McCraty, R., Shaffer, F., 2015. Heart Rate Variability: New Perspectives on Physiological Mechanisms, Assessment of Self-regulatory Capacity, and Health risk. Glob. Adv. Health Med. 4, 46-61.

McKinnon, M.C., Yucel, K., Nazarov, A., MacQueen, G.M., 2009. A meta-analysis examining clinical predictors of hippocampal volume in patients with major depressive disorder. J. Psychiatry Neurosci. 34, 41-54.

Montano, N., Porta, A., Cogliati, C., Costantino, G., Tobaldini, E., Casali, K.R., Iellamo, F., 2009. Heart rate variability explored in the frequency domain: a tool to investigate the link between heart and behavior. Neurosci. Biobehav. Rev. 33, 71-80.

Moon, E., Lee, S.-H., Kim, D.-H., Hwang, B., 2013. Comparative Study of Heart Rate Variability in Patients with Schizophrenia, Bipolar Disorder, Post-traumatic Stress Disorder, or Major Depressive Disorder. Clin. Psychopharmacol. Neurosci. 11, $137-143$.

Ozerdema, A., Güntekind, B., Atagüne, M.I., Bașar, E., 2013. Brain oscillations in bipolar disorder in search of new biomarkers. Suppl. Clin. Neurophysiol. 62, 207-221.

Parker, S.L., Laurie, K.R., Newton, C.J., Jimmieson, N.L., 2014. Regulatory focus moderates the relationship between task control and physiological and psychological markers of stress: a work simulation study. Int. J. Psychophysiol. 94, 390-398.

Patron, E., Messerotti Benvenuti, S., Favretto, G., Valfrè, C., Bonfà, C., Gasparotto, R., Palomba, D., 2012. Association between depression and heart rate variability in patients after cardiac surgery: a pilot study. J. Psychosom. Res. 73, 42-46.

Peterson, J.C., Charlson, M.E., Wells, M.T., Altemus, M., 2014. Depression, coronary artery disease, and physical activity: how much exercise is enough? Clin. Ther. 36, $1518-1530$.

Quintana, D.S., Westlye, L.T., Kaufmann, T., Rustan, Ø.G., Brandt, C.L., Haatveit, B., Steen, N.E., Andreassen, O.A., 2015. Reduced heart rate variability in schizophrenia and bipolar disorder compared to healthy controls. Acta Psychiatr. Scand..

Radaelli, A., Mancia, G., Balestri, G., Rovati, A., Anzuini, A., Di Rienzo, M., Paolini, G., Castiglioni, P., 2014. Cardiovascular variability is similarly altered in coronary patients with normal left ventricular function and in heart failure patients. J. Hypertens. 32, 2261-2266, (discussion 2266).

Rahman, F., Pechnik, S., Gross, D., Sewell, L., Goldstein, D.S., 2011. Low frequency power of heart rate variability reflects baroreflex function, not cardiac sympathetic innervation. Clin. Auton. Res. 21, 133-141.

Rajendra Acharya, U., Paul Joseph, K., Kannathal, N., Lim, C.M., Suri, J.S., 2006. Heart rate variability: a review. Med. Biol. Eng. Comput. 44, 1031-1051.

Reyes del Paso, G.A., Langewitz, W., Mulder, L.J., van Roon, A., Duschek, S., 2013. The utility of low frequency heart rate variability as an index of sympathetic cardiac tone: a review with emphasis on a reanalysis of previous studies. Psychophysiology 50, 477-487.

Rovai, D., Giannessi, D., Andreassi, M.G., Gentili, C., Pingitore, A., Glauber, M., Gemignani, A., 2015. Mind injuries after cardiac surgery. J. Cardiovasc. Med. (Hagerstown, Md. ) 16, 844-851.

Rush, A.J., Trivedi, M.H., Ibrahim, H.M., Carmody, T.J., Arnow, B., Klein, D.N., Markowitz, J.C., Ninan, P.T., Kornstein, S., Manber, R., Thase, M.E., Kocsis, J.H., Keller, M.B., 2003. The 16-Item Quick Inventory of depressive symptomatology (QIDS), clinician rating (QIDS-C), and self-report (QIDS-SR): a psychometric evaluation in patients with chronic major depression. Biol. Psychiatry 54, 573-583.

Salvioli, B., Pellegatta, G., Malacarne, M., Pace, F., Malesci, A., Pagani, M., Lucini, D., 2015. Autonomic nervous system dysregulation in irritable bowel syndrome. Neurogastroenterol. Motil. 
Sanchez-Gonzalez, M.A., Guzik, P., May, R.W., Koutnik, A.P., Hughes, R., Muniz, S., Kabbaj, M., Fincham, F.D., 2014. Trait anxiety mimics age-related cardiovascular autonomic modulation in young adults. J. Hum. Hypertens..

Sandercock, G.R.H., Brodie, D.A., 2006. The role of heart rate variability in prognosis for different modes of death in chronic heart failure. Pacing Clin. Electro. 29, 892-904.

Sartori, G., Pellegrini, S., Mechelli, A., 2011. Forensic neurosciences: from basicresearch to applications and pitfalls. Curr. Opin. Neurol. 24 (4), 371-377.

Saul, J.P., Berger, R.D., Albrecht, P., Stein, S.P., Chen, M.H., Cohen, R.J., 1991. Transfer function analysis of the circulation: unique insights into cardiovascular regulation. Am. J. Physiol. 261, H1231-H1245.

Stapelberg, N.J., Hamilton-Craig, I., Neumann, D.L., Shum, D.H., McConnell, H., 2012. Mind and heart: heart rate variability in major depressive disorder and coronary heart disease - a review and recommendations. Aust. N. Z. J. Psychiatry 46, 946-957.

Tan, C.O., Cohen, M.A., Eckberg, D.L., Taylor, J.A., 2009. Fractal properties of human heart period variability: physiological and methodological implications. J. Physiol. 587, 3929-3941.

Tanev, G., Saadi, D.B., Hoppe, K., Sorensen, H.B.D., 2014. Classification of acute stress using linear and non-linear heart rate variability analysis derived from sternal ECG. Conf. Proc. IEEE Eng. Med Biol. Soc. 2014, 3386-3389.

Tang, S.-C., Jen, H.-I., Lin, Y.-H., Hung, C.-S., Jou, W.-J., Huang, P.-W., Shieh, J.-S., Ho, Y.-L., Lai, D.-M., Wu, A.-Y., Jeng, J.-S., Chen, M.-F., 2015. Complexity of heart rate variability predicts outcome in intensive care unit admitted patients with acute stroke. J. Neurol. Neurosurg. Psychiatry 86, 95-100.

Task Force of the European Society of Cardiology, aT.FotE.So.C., 1996. Heart rate variability: standards of measurement, physiological interpretation and clinical use. task force of the European Society of Cardiology and the North American Society of pacing and Electrophysiology. Circulation 93, 1043-1065.

Teixeira, A.L., Barbosa, I.G., Machado-Vieira, R., Rizzo, L.B., Wieck, A., Bauer, M.E., 2013. Novel biomarkers for bipolar disorder. Expert Opin. Med Diagn. 7, 147-159.

Thayer, J.F., Ahs, F., Fredrikson, M., Sollers, J.J., Wager, T.D., 2012. A meta-analysis of heart rate variability and neuroimaging studies: implications for heart rate variability as a marker of stress and health. Neurosci. Biobehav. Rev. 36, 747-756.

Thayer, J.F., Lane, R.D., 2009. Claude Bernard and the heart-brain connection: further elaboration of a model of neurovisceral integration. Neurosci. Biobehav. Rev. 33, 81-88.

Todder, D., Bersudsky, Y., Cohen, H., 2005. Nonlinear analysis of RR interval in euthymic bipolar disorder. Auton. Neurosci. 117, 127-131.

Udupa, K., Sathyaprabha, T.N., Thirthalli, J., Kishore, K.R., Raju, T.R., Gangadhar, B.N., 2007. Modulation of cardiac autonomic functions in patients with major depression treated with repetitive transcranial magnetic stimulation. J. Affect. Disord. 104 $231-236$
Valenza, G., Citi, L., Gentili, C., Lanata, A., Scilingo, E.P., Barbieri, R., 2014a. Pointprocess nonlinear autonomic assessment of depressive states in bipolar patients. Methods Inf. Med. 53, 296-302.

Valenza, G., Citi, L., Gentili, C., Lanata, A., Scilingo, E.P., Barbieri, R., 2015a. Characterization of depressive States in bipolar patients using wearable textile technology and instantaneous heart rate variability assessment. IEEE J. Biomed. Health Inf. 19, 263-274.

Valenza, G., Gentili, C., Lanatà, A., Scilingo, E.P., 2013. Mood recognition in bipolar patients through the PSYCHE platform: preliminary evaluations and perspectives. Artif. Intell. Med. 57, 49-58.

Valenza, G., Nardelli, M., Lanata, A., Gentili, C., Bertschy, G., Kosel, M., Scilingo, E.P., 2016. Predicting mood changes in bipolar disorder Through heartbeat nonlinear dynamics. IEEE J. Biomed. Health Inform. 20, 1034-1043.

Valenza, G., Nardelli, M., Lanata, A., Gentili, C., Bertschy, G., Paradiso, R., Scilingo, E.P., 2014b. Wearable monitoring for mood recognition in bipolar disorder based on history-dependent long-term heart rate variability analysis. IEEE J. Biomed. Health Inform. 18, 1625-1635.

Valenza, G., Nardelli, M., Lanata, A., Gentili, C., Bertschy, G., Scilingo, E.P., 2015b. Predicting mood changes in bipolar disorder through heartbeat nonlinear dynamics: A preliminary study, Computing in Cardiology Conference (CinC), 2015. IEEE, pp. 801-804.

Wang, Y., Zhao, X., O'Neil, A., Turner, A., Liu, X., Berk, M., 2013. Altered cardiac autonomic nervous function in depression. BMC Psychiatry 13, 187.

Warren, J.H., Jaffe, R.S., Wraa, C.E., Stebbins, C.L., 1997. Effect of autonomic blockade on power spectrum of heart rate variability during exercise. Am. J. Physiol. 273, R495-R502.

Wendt, H., Kiyono, K., Abry, P., Hayano, J., Watanabe, E., Yamamoto, Y., 2014. MultiScale wavelet p-leader based heart rate variability analysis for survival probability assessment in CHF patients. Conf. Proc. IEEE Eng. Med Biol. Soc. 2014, $2809-2812$

Yang, A.C., Huang, C.-C., Yeh, H.-L., Liu, M.-E., Hong, C.-J., Tu, P.-C., Chen, J.-F., Huang, N.E., Peng, C.-K., Lin, C.-P., Tsai, S.-J., 2013. Complexity of spontaneous BOLD activity in default mode network is correlated with cognitive function in normal male elderly: a multiscale entropy analysis. Neurobiol. Aging 34, 428-438.

Yang, A.C., Tsai, S.-J., 2013. Is mental illness complex? From behavior to brain. Prog. Neuropsychopharmacol. Biol. Psychiatry 45, 253-257.

Young, R.C., Biggs, J.T., Ziegler, V.E., Meyer, D.A., 1978. A rating scale for mania: reliability, validity and sensitivity. Br. J. Psychiatry 133, 429-435.

Zhang, L.-N., Yang, G., Cheng, C., Shen, C., Cui, Y.-Y., Zhang, J., Zhang, J.-J., Shen, Z.-X., Zeng, M., Ge, Y.-F., Sun, B., Yu, X.-B., Ouyang, C., Zhang, B., Mao, H.-J., Liu, J., Xing, C.-Y., Zha, X.-M., Wang, N.-N., 2015. Plasma FGF23 levels and heart rate variability in patients with stage 5 CKD. Osteoporos. Int. 26, 395-405. 